\title{
SIMULATION RELATIONS FOR DISCRETE-TIME LINEAR SYSTEMS
}

\author{
Herbert Tanner and George J. Pappas \\ Department of Electrical Engineering \\ University of Pennsylvania \\ Philadelphia, PA 19102 \\ tanneregrasp.cis.upenn.edu, \\ pappasg@ee.upenn.edu
}

\begin{abstract}
Simulation relations of labeled transition systems are used in theoretical computer science in order to formally establish notions of modeling abstraction and refinement in hierarchical systems. In this paper, we establish and characterize simulation relations for arbitrary discrete-time, linear control systems. More precisely, given two discrete-time systems, we consider various embeddings into labeled transition systems, that differ in the amount of timing information that is maintained in the transition relation. For each embedding, we obtain necessary and sufficient conditions for one discrete-time system simulating the transitions of the other. Naturally, the simulation characterizations become weaker as more information is abstracted away in the embedding.
\end{abstract}

Keywords: Transition systems, discrete-time systems, simulation relations, reachability, control invariant subspaces.

\section{INTRODUCTION}

Theoretical computer science, and, in particular, the areas of concurrency theory (Milner, 1989), and computer aided verification (Manna and Pnueli, 1995) have established formal notions of abstraction and model refinement which are used to tackle the exponential explosion arising in the formal analysis and design of purely discrete systems. In the control community, similar notions have been considered in the hierarchical, supervisory control of discrete event systems (Caines and Wei, 1995; Wong and Wonham, 1995), and hybrid systems (see surveys by Alur et al. (2000) and Koutsoukos et al. (2000)).

Simulation relations of labeled transition systems is one such formal notion of abstraction (Milner, 1989). Roughly, transition system $T_{2}$ simulates transition system $T_{1}$, if every transition taken by $T_{1}$ can be matched by a similar transition taken by $T_{2}$. System $T_{2}$ may, however, contain transitions infeasible in $T_{1}$ and thus overapproximates the transitions of $T_{1}$. Simulation re- lations are used in order to establish modeling consistency between various levels of hierarchical systems, as transitions of the coarser (higher level) system $T_{1}$ can be matched by the more complicated (lower level) system $T_{2}$. In the case where $T_{2}$ is smaller in size than $T_{1}$, simulation relations allow reducing the complexity of $T_{1}$, as it is sufficent to analyze $T_{2}$.

As mentioned in van der Schaft and Schumacher (2001), simulation relations have escaped the world of purely continuous systems. More recently, a notion of simulation was introduced for continuous-time systems in Pappas et al. (2000). Given a continuous system and quotient map, a formal construction was provided for extracting quotient systems that simulated the trajectories of the original system. Furthermore, we characterized linear quotient maps that preserve control theoretic properties such as controllability (Pappas et al., 2000), and stabilizability (Pappas and Lafferriere, 2001). Similar results have also been established for nonlinear systems in Pappas and Simic (2001). 
In this paper, we consider for the first time simulation relations for any two discrete-time linear systems in the exact sense that the notion is used in theoretical computer science. More precisely, given any two discrete-time linear control systems, we consider a variety of labeled transition systems that are generated by the linear systems. In particular, we consider timed and time-abstract transition systems generated by linear systems, depending on whether we wish to maintain or ignore timing information as labels on the transitions. In all cases, however, we abstract away control information, in contrast to model reduction results which reduce systems while preserving control input information (Aoki, 1968).

Given two discrete-time systems and the associated embeddings into transition systems, we obtain necessary and sufficient conditions under which one system simulates the transitions of the other. The more information that is abstracted away in the embedding, the weaker the conditions become. In the special case of surjective simulation relations, we obtain as a corollary the discrete-time analogues of the results in Pappas et al. (2000).

In Section 2 we briefly review simulation relations for labeled transition systems. In Section 3, we embedd discrete-time linear systems into various transition systems, and in Section 4 we characterize simulation relations for transition systems generated by linear systems. Section 5 contains various special cases of the main results.

\section{SIMULATIONS OF LABELED TRANSITION SYSTEMS}

In this section we review the standard definitions of simulation relations for transition systems (Milner, 1989).

Definition 2.1. A labeled transition system is a tuple $T=(\mathrm{Q}, \Sigma, \longrightarrow)$ that consists of:

- A (possibly infinite) set $Q$ of states,

- A (possibly infinite) set $\Sigma$ of labels,

- A transition relation $\longrightarrow \subseteq \mathrm{Q} \times \Sigma \times \mathrm{Q}$,

The transition $\left(q_{1}, \sigma, q_{2}\right) \in \longrightarrow$ is commonly denoted as $q_{1} \stackrel{\sigma}{\longrightarrow} q_{2}$. The transition system is called finite is $Q$ and $\Sigma$ are finite, and infinite otherwise. A region is a subset $P \subseteq Q$ of the states. The $\sigma$-successor of a region $P$ is defined as the set that can be reached from $P$ with one $\sigma$-transition. More precisely,

$$
\operatorname{Post}_{\sigma}(P)=\{q \in Q \mid \exists p \in P \text { with } p \stackrel{\sigma}{\longrightarrow} q\}
$$

Simulation relations between transition systems are used to formally define when one transition system implements another.
Definition 2.2. Let $T_{1}=\left(\mathrm{Q}_{1}, \Sigma, \longrightarrow_{1}\right)$ and $T_{2}=\left(\mathrm{Q}_{2}\right.$, $\Sigma, \longrightarrow_{2}$ ) be two transition systems over the same label set $\Sigma$. The relation $S \subseteq \mathrm{Q}_{1} \times \mathrm{Q}_{2}$ is called a simulation relation if for all $\left(q_{1}, q_{2}\right) \in S$, the following property holds:

- if $q_{1} \stackrel{\sigma}{\longrightarrow} q_{1}^{\prime}$, then there exists $q_{2}^{\prime} \in Q_{2}$ with $q_{2} \stackrel{\sigma}{\longrightarrow} q_{2}^{\prime}$ and $\left(q_{1}^{\prime}, q_{2}^{\prime}\right) \in S$

If such a simulation relation exists, then $T_{2}$ simulates (or implements) $T_{1}$, since every transition taken by $T_{1}$ can be matched by $T_{2}$. Note that the label set $\Sigma$ is common to both transition systems. In general $T_{2}$ may have many more transitions, and may be a much more complicated system. Transition system $T_{1}$ can also serve as a more abstract description of transition system $T_{2}$.

In terms of $\sigma$-successors, Definition 2.2 can be rephrased as.

Definition 2.3. Transition system $T_{2}$ simulates $T_{1}$ iff for all $\left(q_{1}, q_{2}\right) \in S$ and for all $\sigma \in \Sigma$ we have

$$
\forall q_{1}^{\prime} \in \operatorname{Post}_{\sigma}\left(q_{1}\right), \exists q_{2}^{\prime} \in \operatorname{Post}_{\sigma}\left(q_{2}\right):\left(q_{1}^{\prime}, q_{2}^{\prime}\right) \in S .
$$

The goal of this paper is to establish simulation relations for linear systems. In order to achieve this, we must relate the world of linear systems with the world of transition systems.

\section{EMBEDDING LINEAR SYSTEMS INTO TRANSITION SYSTEMS}

Every discrete-time linear system can be embedded into various labeled transition systems. We consider various embeddings that differ in the amount of timing information retained or abstracted away on the transitions. For each embedding, the semantics (and eventually the characterization) of the simulation relation will be different.

Consider discrete-time, linear control systems:

$$
\Delta: \quad x_{k+1}=A x_{k}+B u_{k}
$$

with time $k \in \mathbb{N}_{+}$, state $x_{k} \in \mathbb{R}^{n}$, control $u_{k} \in \mathbb{R}^{m}$, and matrices $A, B$ of appropriate dimension. From linear systems theory (Wonham, 1985), we know that given an initial condition $x_{0}$ at time zero, and an input sequence $\left\{u_{i}\right\}_{i=0}^{k-1}=\left\{u_{0}, u_{1}, \ldots, u_{k-1}\right\}$, then the state $x_{k}$ at time $k$ is

$$
x_{k}=A^{k} x_{0}+\sum_{i=0}^{k-1} A^{k-i-1} B u_{i}
$$

The first embedding of discrete-time systems into control systems maintains only transitions that happen in one time step, abstracting away the control that was used for the transition.

Definition 3.1. The one-step transition system $T_{\Delta}^{1}=$ $(Q, \Sigma, \longrightarrow)$ generated by $\Delta$ consists of: 
- State space $Q=\mathbb{R}^{n}$,

- Unique label $\Sigma=\{1\}$,

- Transition relation $\longrightarrow \subseteq Q \times\{1\} \times Q$ with

$$
x \stackrel{1}{\longrightarrow} x^{\prime} \Leftrightarrow \exists u \text { such that } x^{\prime}=A x+B u
$$

The transitions of the one-step transition system naturally correspond to evolution of the discrete-time system in one time step (hence the unique label 1 on the transitions). Furthermore, the transitions of Definition 3.1 are control abstract in the sense that the transition system does not care which particular control $u$ is responsible for the transition of the discrete-time system.

There are two natural variations of Definition 3.1. The first variation maintains not only 1-step transitions, but also $k$-step transitions for any $k \in \mathbb{N}_{+}$, whereas the second variation does not care how many time steps are needed for a transition.

Definition 3.2. The timed transition system $T_{\Delta}^{\mathbb{N}_{+}}=$ $(Q, \Sigma, \longrightarrow)$ generated by $\Delta$ consists of:

- State space $Q=\mathbb{R}^{n}$,

- Label set $\Sigma=\mathbb{N}_{+}$,

- Transition relation $\longrightarrow \subseteq Q \times \mathbb{N}_{+} \times Q$ with

$$
\begin{aligned}
x \stackrel{k}{\longrightarrow} x^{\prime} \Longleftrightarrow & \exists\left\{u_{i}\right\}_{i=0}^{k-1} \text { such that } \\
& x^{\prime}=A^{k} x+\sum_{i=0}^{k-1} A^{k-i-1} B u_{i}
\end{aligned}
$$

More intuitively, there exists a transition $x \stackrel{k}{\longrightarrow} x^{\prime}$ if there is an appropriate sequence of control inputs $\left\{u_{i}\right\}_{i=0}^{k-1}$ that in exactly $k$ time steps will result in the discrete-time system $\Delta$ reaching state $x^{\prime}$ from state $x$.

Contrary to $T^{\mathbb{N}_{+}}$which maintains all timing information, the following transition system does not care about the exact number of time steps needed to reach a state. Since it abstracts away timing information, it is called a time-abstract transition system.

Definition 3.3. The time-abstract transition system $T_{\Delta}^{\tau}=(Q, \Sigma, \longrightarrow)$ generated by $\Delta$ consists of:

- State space $Q=\mathbb{R}^{n}$,

- Unique label $\Sigma=\{\tau\}$,

- Transition relation $\longrightarrow \subseteq Q \times\{\tau\} \times Q$ with

$$
\begin{gathered}
x \stackrel{\tau}{\longrightarrow} x^{\prime} \Longleftrightarrow \exists k \in \mathbb{N}_{+} \exists\left\{u_{i}\right\}_{i=0}^{k-1} \text { such that } \\
x^{\prime}=A^{k} x+\sum_{i=0}^{k-1} A^{k-i-1} B u_{i}
\end{gathered}
$$

In other words, a transition $x \stackrel{\tau}{\longrightarrow} x^{\prime}$ occurs if $x^{\prime}$ is reachable from $x$ in any number of steps by an appropriate sequence of control inputs. Therefore, $T_{\Delta}^{\tau}$ is both time-abstract and control-abstract.

\section{SIMULATION CHARACTERIZATIONS}

Consider two discrete-time linear systems, $\Delta_{1}$ and $\Delta_{2}$ of the form,

$$
\begin{array}{ll}
\Delta_{1} & x_{k+1}=\mathrm{A} x_{k}+\mathrm{B} u_{k}, \quad x \in \mathbb{R}^{n}, u \in \mathbb{R}^{m} \\
\Delta_{2} & z_{k+1}=\mathrm{F} z_{k}+\mathrm{G} v_{k}, \quad z \in \mathbb{R}^{r}, v \in \mathbb{R}^{s}
\end{array}
$$

where $A, B, F$, and $G$ are of appropriate dimension. Both linear systems, $\Delta_{1}$ and $\Delta_{2}$ generate the transition systems described in Section 3. We are now interested in determining necessary and sufficient conditions on the discrete time systems under which $T_{\Delta_{2}}^{1}$ simulates $T_{\Delta_{1}}^{1}, T_{\Delta_{2}}^{\mathbb{N}_{+}}$simulates $T_{\Delta_{1}}^{\mathbb{N}_{+}}$, and $T_{\Delta_{2}}^{\tau}$ simulates $T_{\Delta_{1}}^{\tau}$. Clearly, different embeddings will result in different characterizations.

The simulation relations that we shall consider in this paper are of the form $S \subseteq \mathbb{R}^{n} \times \mathbb{R}^{r}$ where

$$
(x, \mathrm{H} x+y) \in S \subseteq \mathbb{R}^{n} \times \mathbb{R}^{r}, \quad y \in \mathrm{Y}
$$

where $\mathrm{H} \in \mathbb{R}^{r \times n}$ is an arbitrary linear map, and $\mathrm{Y} \subseteq \mathbb{R}^{r}$ is a subspace. The relation $S$ can be regarded as a set valued map assigning to each $x \in \mathbb{R}^{n}$ an affine set $\mathrm{H} x+\mathrm{Y} \subseteq \mathbb{R}^{r}$.

The structure of the relations (6) considered in this paper captures at least two important cases. In the first case, where $Y=0$ and the map $H x$ is surjective, we are interested in simulating the transitions of $\Delta_{1}$ by a system $\Delta_{2}$, which should be smaller in size, thus performing complexity reduction. In the second case, where the map $H x$ is injective and $Y=R(H)^{\perp}$, we are interested in the more complicated system $\Delta_{2}$ simulating the transitions of the simpler system $\Delta_{1}$, thus refining the transitions from the simpler to the more complicated model.

Theorem 4.1. (One-Step Simulation). Consider discrete time systems $\Delta_{1}$ and $\Delta_{2}$ given in equations $(4,5)$, and a relation $S$ of the form (6). Then $T_{\Delta_{2}}^{1}$ simulates $T_{\Delta_{1}}^{1}$ if and only if

$$
\begin{aligned}
\mathscr{R}(\mathrm{HA}-\mathrm{FH})+\mathscr{R}(\mathrm{HB}) \subseteq \mathscr{R}(\mathrm{G})+\mathrm{Y} \\
F \mathrm{Y} \subseteq \mathrm{Y}+\mathscr{R}(\mathrm{G})
\end{aligned}
$$

Proof: By definition, $\Delta_{2}$ one-step simulates $\Delta_{1}$ with respect to the relation $S$ iff $\forall(x, z) \in S$ :

$$
\forall x^{\prime} \in \operatorname{Post}_{1}(x), \exists z^{\prime} \in \operatorname{Post}_{1}(z):\left(x^{\prime}, z^{\prime}\right) \in S .
$$

Given the structure of $S$ we have $\forall(x, z) \in S$ :

$$
\forall x^{\prime} \in \operatorname{Post}_{1}(x), \exists y_{1} \in \mathrm{Y}: z^{\prime}=\mathrm{H} x+y_{1} \in \operatorname{Post}_{1}(z) .
$$

Substituting the Post ${ }_{1}$ operators for the one-step transition systems generated by $\Delta_{1}$ and $\Delta_{2}$, the above becomes: $\forall(x, z) \in S, \forall u \in \mathbb{R}^{m}, \exists y_{1} \in \mathrm{Y}, \exists v \in \mathbb{R}^{s}$ :

$$
\mathrm{F} z+\mathrm{G} v=\mathrm{H}(\mathrm{A} x+\mathrm{B} u)+y_{1}
$$

and since $(x, z) \in S \Leftrightarrow z=\mathrm{H} x+\mathrm{Y}$, then $\forall x \in \mathbb{R}^{n}$ :

$$
\mathrm{F}\left(\mathrm{H}+y_{2}\right)+\mathrm{G} v=\mathrm{H}(\mathrm{A} x+\mathrm{B} u)+y_{1}
$$


Collecting terms and eliminating the quantifiers:

$$
\mathscr{R}(\mathrm{HA}-\mathrm{FH})+\mathscr{R}(\mathrm{HB})+\mathrm{FY}=\mathscr{R}(\mathrm{G})+\mathrm{Y}
$$

which is a necessary and sufficient condition for simulation, derived from the definition. We proceed to show that (7)-(8) are equivalent to (10)

$(\Rightarrow)$ : from condition (8) we get that $\forall y \in \mathrm{Y}, \exists v_{1} \in$ $\mathbb{R}^{s}, \exists y_{1} \in \mathrm{Y}: \mathrm{F} y=\mathrm{G} v_{1}+y_{1}$. Similarly, from (7), $\forall x \in$ $\mathbb{R}^{n}, \forall u \in \mathbb{R}^{m}, \exists v_{2} \in \mathbb{R}^{s}, \exists y_{2} \in \mathrm{Y}:(\mathrm{HA}-\mathrm{FH}) x+\mathrm{HB} u=$ $\mathrm{G} v_{2}+y_{2}$. Adding these equalities yields $\forall x \in \mathbb{R}^{n}, \forall u \in$ $\mathbb{R}^{m}, \forall y \in \mathrm{Y}, \exists v_{1}, v_{2} \in \mathbb{R}^{s}, \exists y_{1}, y_{2} \in \mathrm{Y}$ :

$$
(\mathrm{HA}-\mathrm{FH}) x+\mathrm{HB} u+\mathrm{F} y=\mathrm{G}\left(v_{1}+v_{2}\right)+\left(y_{1}+y_{2}\right)
$$

and since $\mathrm{Y}$ is a subspace we can write that $\forall x \in$ $\mathbb{R}^{n}, \forall u \in \mathbb{R}^{m}, \forall y_{1} \in \mathrm{Y}, \exists v \in \mathbb{R}^{s}, \exists y_{2} \in \mathrm{Y}$ :

$$
(\mathrm{HA}-\mathrm{FH}) x+\mathrm{HB} u+\mathrm{F} y_{1}=\mathrm{G} v+y_{2}
$$

which by eliminating the quantifiers becomes:

$$
\mathscr{R}(\mathrm{HA}-\mathrm{FH})+\mathscr{R}(\mathrm{HB})+\mathrm{FY} \subseteq \mathscr{R}(\mathrm{G})+\mathrm{Y}
$$

$(\Leftarrow)$ : From (10), since $x$, $u$ and $y_{2}$ belong to subspaces we can have:

(1) for $y_{1}=0 \Rightarrow \forall x, \forall u, \exists v, \exists y_{2}:(\mathrm{HA}-\mathrm{FH}) x+$ $\mathrm{HB} u=\mathrm{G} v+y_{2} \Rightarrow \mathscr{R}(\mathrm{HA}-\mathrm{FH})+\mathscr{R}(\mathrm{HB}) \subseteq$ $\mathscr{R}(\mathrm{G})+\mathrm{Y}$,

(2) for $x=0, u=0 \Rightarrow \forall y_{1}, \exists v, \exists y_{2}: F y_{1}=\mathrm{G} v+y_{2} \Rightarrow$ $\mathrm{FY} \subseteq \mathscr{R}(\mathrm{G})+\mathrm{Y}$.

Notice that condition ( 7) relates the system dynamics and control of the two systems in question, whereas condition ( 8 ) is a condition only on system $\Delta_{2}$ and the simulation relation $S$. This is because the dynamics on the $Y$ subspace have meaning only in the the $\Delta_{2}$ system which may contain more states that $\Delta_{1}$.

Since the transitions of $T_{\Delta_{1}}^{1}$ generate the transitions of $T_{\Delta_{1}}^{\mathbb{N}_{+}}$, it should come as no suprise that simulating 1step transitions is necessary and sufficient to simulate $k$-step transitions.

Theorem 4.2. (Timed Simulation). Consider the discrete time systems $\Delta_{1}$ and $\Delta_{2}$ given in equations $(4,5)$, and a relation $S$ of the form (6). Then $T_{\Delta_{2}}^{\mathbb{N}_{+}}$simulates $T_{\Delta_{1}}^{\mathbb{N}_{+}}$iff conditions (7)-(8) are satisfied:

$$
\begin{aligned}
\mathscr{R}(\mathrm{HA}-\mathrm{FH})+\mathscr{R}(\mathrm{HB}) & \subseteq \mathscr{R}(\mathrm{G})+\mathrm{Y} \\
F \mathrm{Y} & \subseteq \mathrm{Y}+\mathscr{R}(\mathrm{G})
\end{aligned}
$$

Proof: By definition, the necessary and sufficient condition for $\Delta_{2}$ to k-step simulate $\Delta_{1}$ with respect to the relation $S$ is $\forall(x, z) \in S, \forall x^{\prime} \in \operatorname{Post}_{k}(x), \exists z^{\prime} \in \operatorname{Post}_{k}(z)$ : $\left(x^{\prime}, z^{\prime}\right) \in S$. After some manipulation and substitution of the Post $_{k}$ operators, this condition expands to:

$$
\begin{array}{r}
\mathscr{R}\left(\mathrm{HA}^{k}-\mathrm{F}^{k} \mathrm{H}\right)+\sum_{i=0}^{k-1} \mathscr{R}\left(\mathrm{HA}^{i} \mathrm{~B}\right)+\mathrm{F}^{k} \mathrm{Y}= \\
\sum_{i=0}^{k-1} \mathscr{R}\left(\mathrm{F}^{k} \mathrm{G}\right)+\mathrm{Y}
\end{array}
$$

We show that (7)-(8) are equivalent to (10):

$(\Rightarrow)$ : The fact that (7)-(8) imply (10) can be shown by induction: if it holds for $p=k$ then

$$
\mathrm{F}^{p+1} \mathrm{~S} \subseteq \mathrm{F}\left(\sum_{i=0}^{p-1} \mathscr{R}\left(\mathrm{F}^{i} \mathrm{G}\right)+\mathrm{Y}\right) \subseteq \sum_{i=0}^{p} \mathscr{R}\left(\mathrm{F}^{i} \mathrm{G}\right)+\mathrm{Y}
$$

On the other hand, rewrite $\sum_{i=0}^{p} \mathscr{R}\left(\mathrm{HA}^{i} \mathrm{~B}\right)$ as $\sum_{i=0}^{p-1}$ $\mathscr{R}\left(\mathrm{HA}^{i} \mathrm{~B}\right)+\mathscr{R}\left(\mathrm{HA}^{p} \mathrm{~B}-\mathrm{F}^{p} \mathrm{HB}+\mathrm{F}^{p} \mathrm{HB}\right)$ and $\mathscr{R}\left(\mathrm{HA}^{p+1}\right.$ $\left.-\mathrm{F}^{p+1} \mathrm{H}\right)$ as $\mathscr{R}\left(\mathrm{HA}^{p+1}-\mathrm{F}^{p+1} \mathrm{H}+\mathrm{F}^{p} \mathrm{HA}-\mathrm{F}^{p} \mathrm{HA}\right)$. Then, by applying the conditions assumed true for $k=p$ and (11), one arrives at:

$\mathscr{R}\left(\mathrm{HA}^{p+1}-\mathrm{F}^{p+1} \mathrm{H}\right)+\sum_{i=0}^{p} \mathscr{R}\left(\mathrm{HA}^{i} \mathrm{~B}\right) \subseteq \sum_{i=0}^{p} \mathscr{R}\left(\mathrm{F}^{i} \mathrm{G}\right)+\mathrm{Y}$

By induction, (11)-(12) hold for every $k$ and therefore imply (10).

$(\Leftarrow)$ : From (10) if follows $: \forall x \in \mathbb{R}^{n}, \forall y_{2} \in \mathrm{Y}, \forall\left\{u_{i}\right\}_{i=0}^{k-1} \in$ $\mathbb{R}^{m}, \exists y_{1} \in \mathrm{Y}, \exists\left\{v_{i}\right\}_{i=0}^{k-1} \in \mathbb{R}^{s}:\left(\mathrm{HA}^{k}-\mathrm{F}^{k} \mathrm{H}\right) x+\sum_{i=0}^{k-1}$ $\mathrm{HA}^{i} \mathrm{~B} u_{i}+\mathrm{F}^{k} y_{2}=\sum_{i=0}^{k-1} \mathrm{~F}^{i} \mathrm{G} v_{i}+y_{1}$. Since $x, u_{i}$ and $y_{2}$ belong to subspaces we can have:

(1) $y_{2}=0 \Rightarrow \forall x, \forall\left\{u_{i}\right\}_{i=0}^{k-1}, \exists\left\{v_{i}\right\}_{i=0}^{k-1}, \exists y_{1}:\left(\mathrm{HA}^{k}-\right.$ $\left.\mathrm{F}^{k} \mathrm{H}\right) x+\sum_{i=0}^{k-1} \mathrm{HA}^{i} \mathrm{~B} u_{i}=\sum_{i=0}^{k-1} \mathrm{~F}^{i} \mathrm{G} v_{i}+y_{1} \Rightarrow \sum_{i=0}^{k-1}$ $\mathscr{R}\left(\mathrm{HA}^{k}-\mathrm{F}^{k} \mathrm{H}\right)+\sum_{i=0}^{k-1} \mathscr{R}\left(\mathrm{HA}^{i} \mathrm{~B}\right) \subseteq \sum_{i=0}^{k-1} \mathscr{R}\left(\mathrm{F}^{i} \mathrm{G}\right)$ $+\mathrm{Y}$

(2) $x=u_{i}=0 \Rightarrow \forall y_{2}, \exists\left\{v_{i}\right\}_{i=0}^{k-1}, \exists y_{1}: \mathrm{F}^{k} y_{2}=\sum_{i=0}^{k-1} \mathrm{~F}^{i} \mathrm{G} v$ $+y_{1} \Rightarrow \mathrm{F}^{k} \mathrm{Y} \subseteq \sum_{i=0}^{k-1} \mathscr{R}\left(\mathrm{F}^{i} \mathrm{G}\right)+\mathrm{Y}$.

and since this is true for all $k$, it should also hold for $k=1$ in which case we have:

$$
\begin{aligned}
\mathscr{R}(\mathrm{HA}-\mathrm{FH})+\mathscr{R}(\mathrm{HB}) & \subseteq \mathscr{R}(\mathrm{G})+\mathrm{Y} \\
\mathrm{FY} & \subseteq \mathscr{R}(\mathrm{G})+\mathrm{Y}
\end{aligned}
$$

In order to obtain the simulation characterizations for the time abstract transition systems, we shall need the following lemmas. By $R(A, B)$, we denote the reachable subspace from the origin, that is $R(A, B)=$ $\operatorname{Im}\left[B A B \ldots A^{n-1} B\right]$.

Lemma 4.3. Let $\mathrm{A} \in \mathbb{R}^{n \times n}, \mathrm{H} \in \mathbb{R}^{s \times n}, \mathrm{~F} \in \mathbb{R}^{r \times r}, \mathrm{G} \in$ $\mathbb{R}^{r \times s}$ be matrices and $\mathrm{G}$ full rank with $s \leq r$. If, $\mathscr{R}(\mathrm{HA}-\mathrm{FH}) \subseteq R(\mathrm{~F}, \mathrm{G})$, then for every $k$, it holds $\mathscr{R}\left(\mathrm{HA}^{k}-\mathrm{F}^{k} \mathrm{H}\right) \subseteq R(\mathrm{~F}, \mathrm{G})$

Proof: It is shown by induction: clearly it holds for $k=$ 1. Assume it holds for $k=p: \exists p, \mathscr{R}\left(\mathrm{HA}^{p}-\mathrm{F}^{p} \mathrm{H}\right) \subseteq$ $R(\mathrm{~F}, \mathrm{G})$. Then since $\mathrm{HA}^{p+1}-\mathrm{F}^{p+1} \mathrm{H}=\mathrm{HA}^{p+1}-$ $\mathrm{F}^{p+1} \mathrm{H}+\mathrm{F}^{p} \mathrm{HA}-\mathrm{F}^{p} \mathrm{HA}=\left(\mathrm{HA}^{p}-\mathrm{F}^{p} \mathrm{H}\right) \mathrm{A}+\mathrm{F}^{p}(\mathrm{HA}-$ $\mathrm{FH})$, it will be that $\mathscr{R}\left(\mathrm{HA}^{p+1}-\mathrm{F}^{p+1} \mathrm{H}\right) \subseteq \mathscr{R}\left(\left(\mathrm{HA}^{p}-\right.\right.$ $\left.\left.\mathrm{F}^{p} \mathrm{H}\right) \mathrm{A}\right)+\mathscr{R}\left(\mathrm{F}^{p}(\mathrm{HA}-\mathrm{FH})\right) \subseteq R(\mathrm{~F}, \mathrm{G})+\mathrm{F}^{p} R(\mathrm{~F}, \mathrm{G})$. 
However, $R(\mathrm{~F}, \mathrm{G})$ is $\mathrm{F}-$ invariant and therefore, $\mathrm{F}^{p} R(\mathrm{~F}, \mathrm{G}) \in R(\mathrm{~F}, \mathrm{G})$. Thus, $\mathscr{R}\left(\mathrm{HA}^{p+1}-\mathrm{F}^{p+1} \mathrm{H}\right) \subseteq$ $R(\mathrm{~F}, \mathrm{G})$ and therefore by induction, $\forall k, \mathscr{R}\left(\mathrm{HA}^{k}-\right.$ $\left.\mathrm{F}^{k} \mathrm{H}\right) \subseteq R(\mathrm{~F}, \mathrm{G})$

Lemma 4.4. Let $\mathrm{Y}$ be a subspace and $\mathrm{F} \in \mathbb{R}^{r \times r}, \mathrm{G} \in$ $\mathbb{R}^{r \times s}$ be matrices and $\mathrm{G}$ full rank with $s \leq r$. If $\mathrm{FY} \subseteq$ $R(\mathrm{~F}, \mathrm{G})$, then for every $k$, it holds $\mathrm{F}^{k} \mathrm{Y} \subseteq R(\mathrm{~F}, \mathrm{G})$

Proof: It is shown by induction: obviously, it is true for $k=1$. Suppose there exists a $p$ for which: $\mathrm{F}^{p} \mathrm{Y} \subseteq R(\mathrm{~F}, \mathrm{G})$. Then for $k=p+1, \mathrm{~F}^{p+1} \mathrm{Y}=\mathrm{FF}^{p} \mathrm{Y} \subseteq$ $\mathrm{F} R(\mathrm{~F}, \mathrm{G})$, and since $R(\mathrm{~F}, \mathrm{G})$ is $\mathrm{F}-$ invariant, $\mathrm{F}^{p+1} \mathrm{Y} \subseteq$ $R(\mathrm{~F}, \mathrm{G})$. By induction we conclude that $\forall k \in \mathbb{N}_{+}, \mathrm{F}^{k} \mathrm{Y} \subseteq$ $R(\mathrm{~F}, \mathrm{G})$.

Theorem 4.5. (Time-Abstract Simulation). Consider the discrete time systems $\Delta_{1}$ and $\Delta_{2}$ given in equations (4, 5 ), and consider a relation $S$ of the form (6). Then $T_{\Delta_{2}}^{\tau}$ simulates $T_{\Delta_{1}}^{\tau}$ if and only if

$$
\begin{gathered}
\mathscr{R}(\mathrm{HA}-\mathrm{FH})+\mathrm{H} R(\mathrm{~A}, \mathrm{~B}) \subseteq R(\mathrm{~F}, \mathrm{G})+\mathrm{Y} \\
\mathrm{FY} \subseteq \mathrm{Y}+R(\mathrm{~F}, \mathrm{G}) .
\end{gathered}
$$

Proof: By definition, for $\Delta_{2}$ to simulate $\Delta_{1}$ in abstract time, with respect to $S$ it should hold that for every $(x, z) \in S \Rightarrow \forall x^{\prime} \in \operatorname{Post}(x), \exists z^{\prime} \in \operatorname{Post}(z):\left(x^{\prime}, z^{\prime}\right) \in S$ Taking to account the structure of $S$ and the expression for the Post operators: $\forall x \in \mathbb{R}^{n}, \mathrm{H} \bigcup_{k \geq 0} \mathrm{~A}^{k} x+$ $\mathrm{H} R(\mathrm{~A}, \mathrm{~B})+\bigcup_{k \geq 0} \mathrm{~F}^{k}(\mathrm{H} x+\mathrm{Y}) \subseteq R(\mathrm{~F}, \mathrm{G})+\mathrm{Y}$. Collecting terms and eliminating the quantifiers, $\bigcup_{k \geq 0} \mathscr{R}\left(\mathrm{HA}^{k}-\right.$ $\left.\mathrm{F}^{k} \mathrm{H}\right)+\mathrm{H} R(\mathrm{~A}, \mathrm{~B})+\bigcup_{k>0} \mathrm{~F}^{k} \mathrm{Y} \subseteq R(\mathrm{~F}, \mathrm{G})+\mathrm{Y}$. By lemmas 4.3 and 4.4 this reduces to:

$$
\mathscr{R}(\mathrm{HA}-\mathrm{FH})+\mathrm{H} R(\mathrm{~A}, \mathrm{~B})+\mathrm{FY} \subseteq R(\mathrm{~F}, \mathrm{G})+\mathrm{Y}
$$

Equation (15) is necessary and sufficient for $\Delta_{2}$ to simulate $\Delta_{1}$ in abstract time. It remains to establish the equivalence of (13)-(14) to (15):

$(\Rightarrow)$ : from condition (14) we get that $\forall y \in \mathrm{Y}, \exists v_{1} \in$ $\mathbb{R}^{s}, \exists y_{1} \in \mathrm{Y}: \mathrm{F} y=\mathrm{G} v_{1}+y_{1}$. Similarly, from (13), $\forall x \in$ $\mathbb{R}^{n}, \forall w \in R(\mathrm{~A}, \mathrm{~B}), \exists v_{2} \in \mathbb{R}^{s}, \exists y_{2} \in \mathrm{Y}:(\mathrm{HA}-\mathrm{FH}) x+$ $\mathrm{HB} w=\mathrm{G}_{2}+y_{2}$. Adding these equalities yields $\forall x \in$ $\mathbb{R}^{n}, \forall w \in R(\mathrm{~A}, \mathrm{~B}), \forall y \in \mathrm{Y}, \exists v_{1}, v_{2} \in \mathbb{R}^{s}, \exists y_{1}, y_{2} \in \mathrm{Y}:$

$$
(\mathrm{HA}-\mathrm{FH}) x+\mathrm{H} w+\mathrm{F} y=\mathrm{G}\left(v_{1}+v_{2}\right)+\left(y_{1}+y_{2}\right)
$$

and since $\mathrm{Y}$ is a subspace we have $\forall x \in \mathbb{R}^{n}, \forall w \in$ $R(\mathrm{~A}, \mathrm{~B}), \forall y_{1} \in \mathrm{Y}, \exists v \in \mathbb{R}^{s}, \exists y_{2} \in \mathrm{Y}$ :

$$
(\mathrm{HA}-\mathrm{FH}) x+\mathrm{H} w+\mathrm{F} y_{1}=\mathrm{G} v+y_{2}
$$

which by eliminating the quantifiers becomes:

$$
\mathscr{R}(\mathrm{HA}-\mathrm{FH})+\mathrm{H} R(\mathrm{~A}, \mathrm{~B})+\mathrm{FY} \subseteq \mathscr{R}(\mathrm{G})+\mathrm{Y}
$$

$(\Leftarrow)$ : From (15), since $x \in \mathbb{R}^{n}, w \in R(\mathrm{~A}, \mathrm{~B})$ and $y_{2} \in \mathrm{Y}$ belong to subspaces we have:
(1) for $y_{1}=0 \Rightarrow \forall x, \forall w \in R(\mathrm{~A}, \mathrm{~B}), \exists v, \exists y_{2}$ : (HA $\mathrm{FH}) x+\mathrm{H} w=\mathrm{G} v+y_{2} \Rightarrow \mathscr{R}(\mathrm{HA}-\mathrm{FH})+\mathrm{H} R(\mathrm{~A}, \mathrm{~B})$ $\subseteq \mathscr{R}(\mathrm{G})+\mathrm{Y}$

(2) for $x=0, w=0 \Rightarrow \forall y_{1}, \exists v, \exists y_{2}: F y_{1}=\mathrm{G} v+y_{2} \Rightarrow$ $\mathrm{FY} \subseteq \mathscr{R}(\mathrm{G})+\mathrm{Y}$.

It is straightfoward that the simulation conditions $(13,14)$ in the time-abstract case are clearly weaker that their counterparts $(7,8)$ in the timed-simulation case. Therefore, as timing information is abstracted away from the semantics of the transition systems, the simulation conditions become weaker.

\section{SPECIAL CASES}

Simulation relations induce partial order relations among transition systems. In the case where transition system $T_{2}$ simulates transition system $T_{1}$ with simulation relation $S$ and, in addition, $T_{1}$ simulates $T_{2}$ with relation $S^{-1}$, then $T_{1}$ and $T_{2}$ are called bisimilar.

Consider now two discrete-time systems and a relation $S$ of the form

$$
(x, H x) \in S \subseteq \mathbb{R}^{n} \times \mathbb{R}^{r}
$$

where $\mathrm{H} x$ is a surjective map (that is $r<n$ ). The map $z=\mathrm{H} x$ is therefore grouping states in $\mathbb{R}^{n}$ to a single state in $\mathbb{R}^{r}$, thus inducing a partition of $\mathbb{R}^{n}$. This partition aims at complexity reduction from the original system $\Delta_{1}$ to the quotient system $\Delta_{2}$.

The results of the former section can be restated for this special case in the following corollaries.

Corollary 5.1. Transition system $T_{\Delta_{2}}^{1}\left(T_{\Delta_{2}}^{\mathbb{N}_{+}}\right)$simulates $T_{\Delta_{1}}^{1}$ (respectively $T_{\Delta_{1}}^{\mathbb{N}_{+}}$) with simulation relation $S=$ $\left\{(x, z) \in \mathbb{R}^{n} \times \mathbb{R}^{r} \mid z=\mathrm{H} x\right\}$ if and only if

$$
\mathscr{R}(\mathrm{HA}-\mathrm{FH})+\mathscr{R}(\mathrm{HB}) \subseteq \mathscr{R}(\mathrm{G})
$$

Condition (16) is the discrete-time analogue of the notion of $H$-related control systems, first introduced in Pappas et al. (2000). Given system $\Delta_{1}$ with matrices $A, B$, and surjective map $z=H x$, we can always construct system $\Delta_{2}$ and matrices $F, G$, so that condition (16) is satisfied. Since $H$ is full-row rank, one such choice is

$$
\begin{aligned}
& F=H A H^{+} \\
& G=\left[\begin{array}{ll}
H B & H A K \operatorname{Ker}(H)
\end{array}\right]
\end{aligned}
$$

With the above choice of matrices, transition system $T_{\Delta_{2}}^{1}$ simulates $T_{\Delta_{1}}^{1}$. In order for $T_{\Delta_{2}}^{1}$ to be bisimilar to $T_{\Delta_{2}}^{1}, T_{\Delta_{2}}^{1}$ must also simulate $T_{\Delta_{1}}^{1}$ with relation $S^{-1}$, which has the form

$$
\left(z, H^{+} z+\operatorname{Ker}(H)\right) \in S^{-1} \subseteq \mathbb{R}^{r} \times \mathbb{R}^{n}
$$

Therefore, in this case, if we apply conditions $(7,8)$ with $Y=\operatorname{Ker}(H)$ (and the roles of $A, B, H$ interchanged 
with those of $F, G, H^{+}$) we obtain that in order for $T_{\Delta_{2}}^{1}$ to be bisimilar to $T_{\Delta_{1}}^{1}$ it is necessary that

$$
\mathrm{AKer}(H) \subseteq \operatorname{Ker}(H)+\mathscr{R}(\mathrm{B})
$$

In straightforward to show that for the choice of matrices $(17,18)$ this condition is the only relevant necessary and sufficient condition, as (7) is automatically satisfied.

In the time abstract case the relation naturally loosens up since timing information is irrelavant and simulation actually involves aligning the reachable sets of the systems in question.

Corollary 5.2. Transition system $T_{\Delta_{2}}^{\tau}$ simulates $T_{\Delta_{1}}^{\tau}$ with simulation relation $S=\left\{(x, z) \in \mathbb{R}^{n} \times \mathbb{R}^{r} \mid z=\mathrm{H} x\right\}$ if and only if

$$
\mathscr{R}(\mathrm{HA}-\mathrm{FH})+\mathrm{H} R(\mathrm{~A}, \mathrm{~B}) \subseteq R(\mathrm{~F}, \mathrm{G}) .
$$

\section{CONCLUSIONS}

In this paper we characterized simulation relations for various transition systems generated by discrete-time linear systems. The characterizations can be extended to continuous-time systems, as well as to nonlinear and hybrid systems for various transition system semantics.

Of particular interest is to consider such questions for discrete-time systems with state and input constraints. This will allow the not only establishing consistency between different models, but also between their respective constraint sets.

Acknowledgment: The authors would like to thanks Paulo Tabuada for may interesting discussions on the subject. This research is partially supported by DARPA ITO MoBIES Grant F33615-00-C-1707, and the University of Pennsylvania Research Foundation.

\section{REFERENCES}

Alur, R., T. Henzinger, G. Lafferriere and G.J. Pappas (2000). Discrete abstractions of hybrid systems. Proceedings of the IEEE 88(7), 971-984.

Aoki, M. (1968). Control of large scale dynamic systems by aggregation. IEEE Transactions on Automatic Control 13(3), 246-253.

Caines, P.E. and Y.J. Wei (1995). The hierarchical lattices of a finite state machine. Systems and Control Letters 25, 257-263.

Koutsoukos, X., P. Antsaklis, J.Stiver and M. Lemmon (2000). Supervisory control of hybrid systems. Proceedings of the IEEE 88(7), 1026-1049.

Manna, Z. and A. Pnueli (1995). Temporal Verification of Reactive Systems: Safety. Springer Verlag. New York.

Milner, R. (1989). Communication and Concurrency. Prentice Hall.
Pappas, G. J. and G. Lafferriere (2001). Hierarchies of stabilizability preserving linear systems. In: Proceedings of the 40th IEEE Conference in Decision and Control. Orlando, FL.

Pappas, G. J. and S. Simic (2001). Consistent abstractions of affine control systems. IEEE Transactions on Automatic Control. Submitted.

Pappas, G. J., G. Lafferriere and S. Sastry (2000). Hierarchically consistent control systems. IEEE Transactions on Automatic Control 45(6), 11441160.

van der Schaft, A. J. and J. M. Schumacher (2001). Compositionality issues in discrete, continuous, and hybrid systems. International Journal of Robust and Nonlinear Control 11(5), 417-434.

Wong, K.C. and W.M. Wonham (1995). Hierarchical control of discrete-event systems. Discrete Event Dynamic Systems 6, 241-273.

Wonham, W.M. (1985). Linear Multivariable Control : A Geometric Approach. Vol. 10 of Applications of Mathematics. Springer-Verlag. New York. 